\title{
Partial anomalous pulmonary venous connection with anomalous connection of the superior vena cava to the left atrium
}

\author{
Can Yerebakan, MD, ${ }^{\mathrm{a}}$ Lowell Frank, MD, ${ }^{\mathrm{b}}$ Narutoshi Hibino, MD, ${ }^{\mathrm{a}}$ and Richard A. Jonas, MD, ${ }^{\mathrm{a}}$ \\ Washington, DC
}

Partial anomalous pulmonary venous connection (PAPVC) is a very rare congenital anomaly that was first described by Winslow in 1789. Pathologic studies suggest that this anomaly occurs in $0.7 \%$ of the population, ${ }^{1,2}$ which is a higher rate than reported in clinical series. Most cases are associated with a sinus venosus defect. PAPVC from the right lung is twice as common as PAPVC from the left lung. Most commonly, the anomalous connection is to the right superior vena cava (SVC) or the right atrium. Isolated PAPVC with intact atrial septum is even less common and usually involves drainage of the right upper pulmonary vein into the SVC. ${ }^{3}$

In this report, we present a case in which preoperative echocardiography suggested a superior sinus venosus atrial septal defect with anomalous drainage of the right upper lobe to the SVC. Intraoperatively, no atrial septal defect was found. An anomalous vessel was attached to the SVC at the level of multiple small anomalous right upper pulmonary veins to the SVC and was connecting the SVC to the roof of the left atrium. Surgical correction was established by disconnection of the right PAPVC and the anomalous vessel from the SVC, with reconstitution of the SVC by direct anastomosis.

\section{CLINICAL SUMMARY}

A term female neonate was noted to have cyanosis at rest. Echocardiography revealed situs solitus with normal ventricular looping and normally related great vessels. The SVC was thought to be draining to the left atrium through a superior sinus venosus septal defect. The right upper pulmonary vein could not be clearly delineated, although the remainder of the pulmonary veins had normal connections to the left atrium. The SVC was mildly dilated. The patient was followed up as an outpatient and had no cardiac symptoms other than mild cyanosis. She showed normal growth and development.

\footnotetext{
From the Departments of Cardiovascular Surgery ${ }^{\mathrm{a}}$ and Cardiology, ${ }^{\mathrm{b}}$ Children's National Heart Institute, Children's National Medical Center, Washington, DC. Disclosures: Authors have nothing to disclose with regard to commercial support. Received for publication Oct 28, 2011; revisions received Jan 27, 2012; accepted for publication March 12, 2012; available ahead of print April 9, 2012.

Address for reprints: Can Yerebakan, MD, Cardiovascular Surgery, Children's

National Heart Institute, Children's National Medical Center, 111 Michigan Ave

NW, Washington, DC 20010 (E-mail: can.yerebakan@med.uni-rostock.de).

J Thorac Cardiovasc Surg 2012;144:e1-3

$0022-5223 / \$ 36.00$

Copyright (c) 2012 by The American Association for Thoracic Surgery

doi:10.1016/j.jtcvs.2012.03.020
}

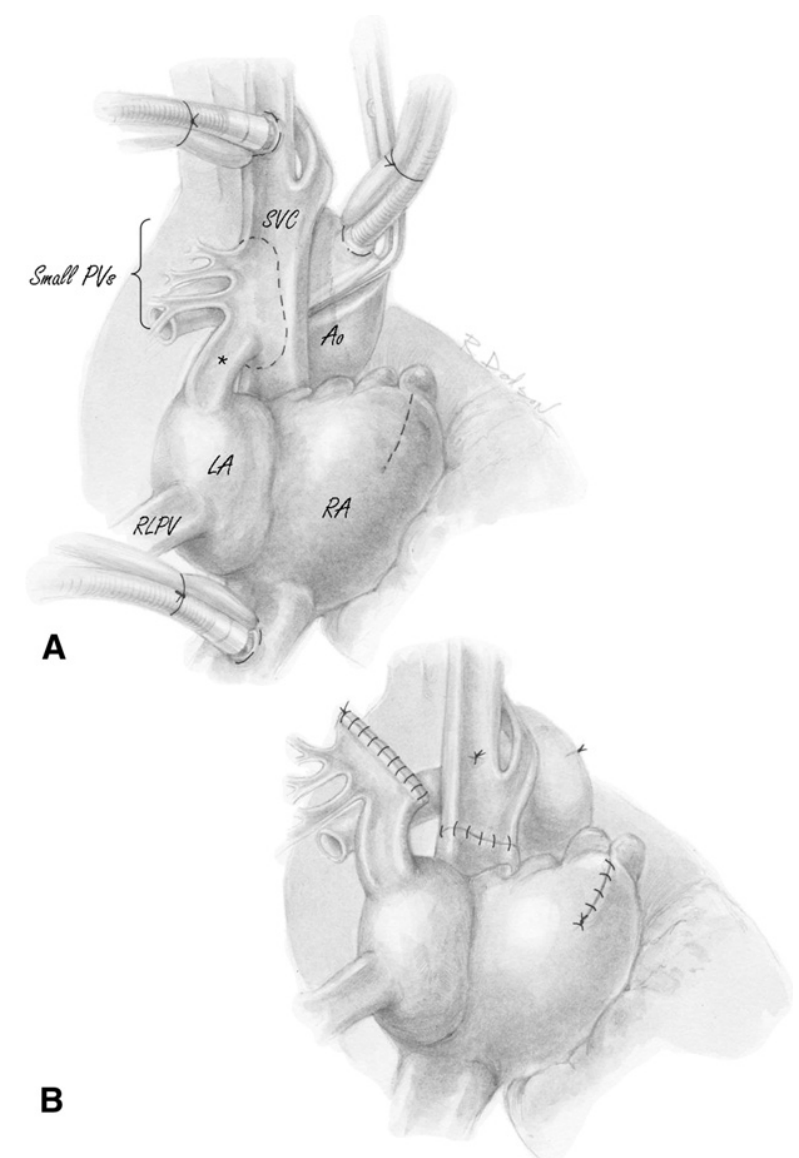

FIGURE 1. A, Anomalous small multiple right upper pulmonary veins $(P V s)$ connect to the superior vena cava $(S V C)$. There is a normal connection of the right lower pulmonary vein $(R L P V)$ to the left atrium $(L A)$. The asterisk marks the large auxiliary superior vena cava to left atrium connection. Dashed lines show surgical incisions on the right atrium $(R A)$ and superior vena cava. B, A procedure including some elements of a Warden procedure was undertaken to separate the anomalous pulmonary veins from the superior vena cava, allowing pulmonary venous blood to flow into the left atrium through the auxiliary connection. The distal superior vena cava was reconstituted by direct anastomosis. Ao, Aorta.

Because of the persistent hypoxia, with peripheral arterial saturations of approximately $90 \%$, the decision for an operative procedure was made when the patient was 1 year old. After partial thymectomy and opening of the pericardium, an anomalous pulmonary venous connection of the right upper lobe to the posterolateral aspect of the SVC was noted. There were multiple small veins from the right upper lobe to the SVC. In addition, there was an 


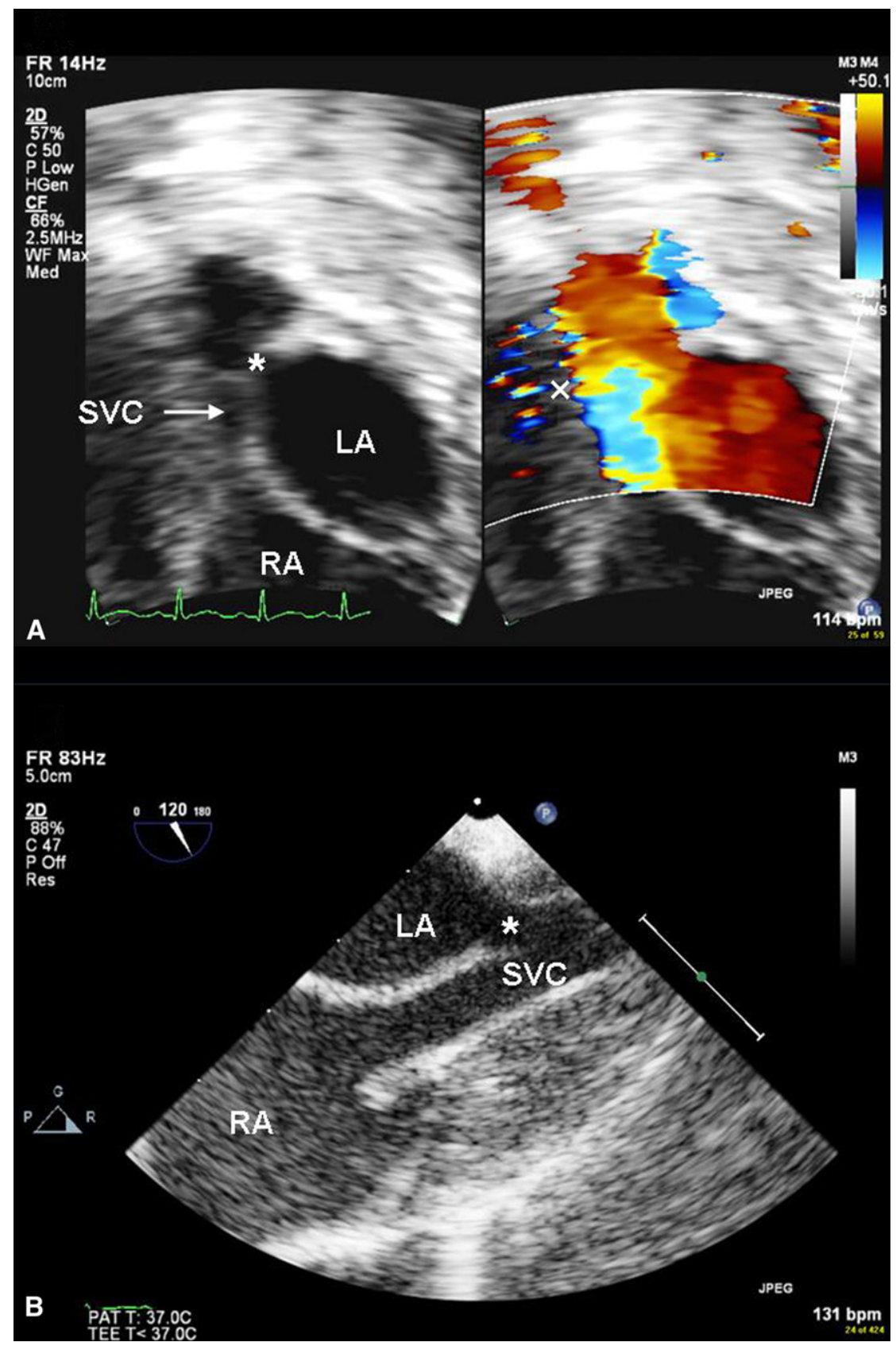

FIGURE 2. A, Transthoracic echocardiographic subcostal view in 2 dimensions and color showing the anomalous connection of the superior vena cava $(S V C)$ to the left atrium $(L A)$. This was initially interpreted a high superior sinus venosus type septal defect. Note the flow acceleration (denoted by times sign) in the distal superior vena cava, which is probably secondary to anomalous pulmonary venous drainage. B, Transesophageal echocardiographic sagittal view showing the anomalous connection between the superior vena cava and the left atrium. Asterisk indicates the anomalous vein between superior vena cava and the left atrium. $R A$, Right atrium.

anomalous vessel the same size as the SVC at the level of the anomalous pulmonary veins, which connected the SVC to the roof of the left atrium (Figures 1, A, and 2).

After heparinization, cardiopulmonary bypass was established with the arterial cannula in the aorta and 2 venous cannulas, 1 in the inferior vena cava and 1 in the left innominate vein. After cardioplegic arrest was established at $28^{\circ} \mathrm{C}$, a clamp was placed across the SVC below the level of the azygos vein. The azygos vein was large and was left patent. The right atrium was opened with a standard short, oblique incision. The SVC was confirmed to be normally connected to the right atrium. There was no atrial septal defect except for a patent foramen ovale. The SVC was transected above and below the anomalous pulmonary veins 
and the anomalous SVC to left atrium vessel. The proximal and distal open ends of the SVC above and below the pulmonary vein orifices were closed by direct suturing with 6/0 Prolene suture (Ethicon, Inc, Somerville, NJ), thus allowing the anomalous veins to drain to the left atrium through the anomalous vessel from the SVC to the left atrium. The SVC was reconstituted by direct anastomosis. The patent foramen ovale was closed by direct suture (Figure 1, B). Weaning from cardiopulmonary bypass was accomplished without problems. Postoperative echocardiography showed no shunt at the SVC or the atrial level, good biventricular function, and no obstruction of pulmonary venous return. The patient was transferred to the cardiac intensive care unit and was discharged home on the 4 th postoperative day.

\section{DISCUSSION}

This is the second case report in the English-language literature to describe isolated PAPVC with concomitant anomalous connection of the SVC to the left atrium. ${ }^{4}$ The surgical repair consisted of a direct complete repair. The unobstructed PAPVC to the SVC could be directed from the SVC to the left atrium through the auxiliary connection. The remainder of the procedure consisted of a direct SVC to right atrial connection without the need for a removal of the tip of the atrial appendage.

Preoperative diagnosis of isolated PAPVR may be difficult because of lung overlying the pulmonary veins and the
SVC. Where there is particular doubt, magnetic resonance imaging (MRI) may be considered for supplementary diagnosis. Although standard evaluation with T1-weighted spin echo images can delineate the exact anatomy, cine-MRI with flow-sensitive sequences may reveal information about the exact size, course, and drainage of the PAPVR. Furthermore, 3-dimensional imaging can be created with magnetic resonance angiography. ${ }^{3}$ If the echocardiographic images are easy to obtain and there is no urgent need, however, MRI is usually not preferred in the younger patient population because of the risks associated with sedation and intubation. Cardiac catheterization is rarely necessary.

In conclusion, even if an accurate preoperative evaluation with echocardiography, MRI, or both has been performed, careful understanding of the entire cardiac anatomy should be achieved intraoperatively in patients with PAPVC to allow application of an appropriate surgical repair.

\section{References}

1. Hughes C, Rumore P. Anomalous pulmonary veins. Arch Pathol. 1944;37:364-6.

2. Healey JE Jr. An anatomic survey of anomalous pulmonary veins: their clinical significance. J Thorac Surg. 1952;23:433-44.

3. Geva T, Van Praagh S. Anomalies of the pulmonary veins. In: Allen HD Driscoll DJ, Shaddy RE, Feltes TF, eds. Moss and Adams' heart diseases in infants, children, and adolescents. 7th ed. Philadelphia: Lippincott Williams \& Wilkins; 2008. p. 761-92.

4. Beck C, Wessel A, Yelbuz TM, Bertram H. Images in cardiovascular medicine Unusual case of anomalous pulmonary venous return with left atrial to systemic venous shunt. Circulation. 2006;113. e840-1.

\title{
Cardiac extension of an intravascular leiomyomatosis 43 years after hysterectomy
}

\author{
François Cornélis, MD, ${ }^{\mathrm{a}}$ Geneviève Belleannée, MD, ${ }^{\mathrm{b}}$ and Mathieu Lederlin, MD, ${ }^{\mathrm{a}}$ Pessac, France
}

Intravenous leiomyomatosis is a rare benign smooth muscle tumor most frequently seen in premenopausal women. Extension through the inferior vena cava into the right heart chambers can cause valve obstruction, leading to cardiac insufficiency and death. Definitive treatment is surgical; however, recurrence from incomplete excision may

\footnotetext{
From the Departments of Thoracic and Cardiovascular Imaging ${ }^{\mathrm{a}}$ and Pathology, Hôpital Haut-Lévèque, CHU Bordeaux, Pessac, France.

Disclosures: Authors have nothing to disclose with regard to commercial support.

Received for publication Feb 1, 2012; accepted for publication March 12, 2012; available ahead of print April 9, 2012.

Address for reprints: Mathieu Lederlin, MD, Department of Thoracic and Cardiovascular Imaging, Hôpital Haut-Lévèque, CHU Bordeaux, 33604 Pessac, France (E-mail: mathieu.lederlin@chu-bordeaux.fr).

J Thorac Cardiovasc Surg 2012;144:e3-5

$0022-5223 / \$ 36.00$

Copyright (c) 2012 by The American Association for Thoracic Surgery

doi:10.1016/j.jtcvs.2012.03.022
}

necessitate reoperation. Intravenous leiomyomatosis should therefore be considered in the differential diagnosis of a middle-aged woman with a right heart tumor.

\section{CLINICAL SUMMARY}

A 75-year-old woman was referred to our institution with swollen legs. She had a history of a hysterectomy and a bilateral ovariectomy at the age of 32 years for a myomatous uterus. Three years before the referral, the patient underwent a surgical resection of a left-sided pelvic tumor that was noted to be a leiomyoma. In the postoperative period, a left internal iliac vein and inferior vena cava thrombosis was diagnosed, and she received anticoagulation therapy for this condition.

Physical examination showed swollen legs without any other abnormality. Cardiac auscultation revealed a tricuspid 